Cite this: DOI: $10.1039 / \mathrm{c} 4 \mathrm{np} 00130 \mathrm{c}$

\title{
Natural products with therapeutic potential in melanoma metastasis
}

\author{
A. AIQathama ${ }^{a, b}$, J.M. Prieto, ${ }^{a^{*}}$
}

Received 08th October 2014,

Accepted 00th

Covered: up to 2014

DOI: $10.1039 / c 4 n p 00130 c$

www.rsc.org/npr

\begin{abstract}
Malignant melanoma is the most aggressive form of skin cancer and accounts for about $3 \%$ of all cases of malignant tumour. Its incidence is increasing worldwide and it is becoming resistant to current therapeutic agents. Natural products continue to provide lead cytotoxic compounds for cancer treatment but less attention has been given to antimigratory compounds. This paper systematically and critically surveys all natural products with direct in vitro and in vivo pharmacological effects on migration and/or metastasis of melanoma cells and maps the mechanisms of action for these underexploited properties. As a result, over 30 natural active principles are described acting mainly through their antagonistic effects upon the TNF- $\alpha$ and EP2 receptors or the suppression of several protein kinases involved in metastatic pathways such as RAS, PI3K, ERK and FAK. Also, some were able to reduce the level of mesenchymal biomarkers such as $\mathrm{N}$-cadherin and/or elevate the expression of other molecules such as E-cadherin. Consequently, downstream transcription factors namely NF-kB, AP-1, ATF-2, CREB, and HIF were inactivated leading to diminished production of MMPs, IL-1, IL-6, COX-2, VEGF and GMCSF. This review also discusses the opportunity of combination therapies based on natural products and approved drugs, such as the combination of EGCG and dacarbazine, or the combination of two natural compounds such as quercetin and sulforaphane.
\end{abstract}

\section{Introduction}

2. Tumour metastasis

2.1 Cell extension

2.2 Adhesion

2.3 Secretion of surface protease and contraction

2.4. Detachement

3. Melanoma and anti-migratory natural products

3.1 Alkaloids

3.2 Polyphenols

3.3 Vitamin-related compounds

3.4 Terpenoids

3.5 Peptides

3.6 Herbal extracts

4. Concluding remarks

5. References

a. Department of Pharmaceutical and Biological Chemistry, UCL School of Pharmacy, 29-39 Brunswick Square, WC1N 1AX London, United Kingdom.Email: j.prieto@ucl.ac.uk

b. Department of Pharmacognosy, Faculty of Pharmacy, Umm Al-Qura University, Makkah, 21955, Saudi Arabia

\section{Introduction}

Melanoma is a highly resistant and malignant cancer accounting for about $3 \%$ of all cases of malignant tumours. ${ }^{1}$ Its incidence has increased faster than that of any other cancer type during recent decades. Although melanoma accounts for only $4 \%$ of all skin cancer cases, it is the most aggressive and causes approximately $80 \%$ of all deaths from skin cancer. ${ }^{2}$ Malignant melanoma has been described as a sequential process that originates from an atypical benign nevus followed by anomalous growth and dysplasia, resulting in a pre-malignant lesion. Malignant melanocytes will then enter into radial growth phase by proliferating horizontally within the epidermis. This is followed by a vertical growth phase, where malignant cells acquire the ability to invade the basement membrane thus entering other tissues. $^{3}$

Although malignant melanoma is considered a multifactorial disease, the major factor contributing to its development is excessive ultraviolet (UV) light exposure. ${ }^{3}$ Sun exposure may induce DNA damage, gene mutation and inflammatory responses, all together contributing to the increased risk of skin cancer. When skin is exposed to UV radiation, cyclooxygenase-2 (COX-2), an inducible enzyme generating prostaglandins (PGs) is activated. $\mathrm{PGE}_{2}$ binds to its G-protein-coupled receptors, EP1, EP2, EP3 and EP4, resulting in angiogenesis, decreased host immunity, enhanced cancer invasion and metastasis. 4 
Ms. Aljawharah AlQathama obtained a Bachelor degree from Umm Al-Qura University (Makkah, Saudi Arabia) in 2006, after which she worked in its Pharmacognosy Department as Teaching Assistant for four years. In 2011 she obtained a scholarship from Ministry of Higher Education in Saudi Arabia to study an MSc degree in Pharmacognosy at the UCL School of Pharmacy (London, United Kingdom), where she graduated with distinction and received the Schwabe Award. She is currently a PhD candidate at the Department of Pharmaceutical and Biological Chemistry (UCL School of Pharmacy) under the supervision of Dr Jose M. Prieto and Prof. Simon Gibbons and working in close collaboration with Umm AlQura University. Her research focuses on the isolation, elucidation and evaluation of the cytotoxic and anti-metastatic properties of natural products in melanoma.

In this review, we first present the reader with a succinct overview of the most important biomolecular pathways in tumour metastasis, followed by a detailed discussion of the activities of more than 30 natural products upon these targets. Other natural products acting through unknown mechanisms of action are briefly discussed together at the end of each section.

\section{Tumour metastasis}

Tumour cells have to undergo a remarkable cascade of events in order to progress from a benign melanocyte hyperplasia into a metastatic melanoma. First, a series of changes in cell motility genes will allow the melanoma cell to coordinate the extension, adhesion, contraction and detachment necessary to move and leave its primary site (Fig. 1). ${ }^{5}$ Once this is achieved, the invasion of surrounding tissues needs a further series of genetic events in order to detach from the primary tumour (dissociation), gain access to the lymphatic vessels (intravasation) and then enter the target organs (extravasation). ${ }^{6,7}$ All this sequence of events is also orchestrated by external factors, including chemokines, proteases, extracellular matrix proteins and growth factors/receptors, integrins, matrixdegrading enzymes, cell-cell adhesion molecules and cell-cell communication proteins. ${ }^{5,6,7,8}$

FIGURE 1 MAY GO HERE (SEE AT THE END OF THE DOCUMENT)

\subsection{Cell extension}

The migration process starts with the extension of a lamellipodium, a protrusion at the leading edge of the cell (Fig. 1a). ${ }^{5}$ This is underpinned by actin polymerization that provides the traction force pushing the membrane forward in the direction of the movement. This is stimulated and regulated by the Rac protein, which belongs to Rho GTPase family. In metastatic tumour cells, this signalling pathway is overactive. ${ }^{9}$

\subsection{Adhesion}

Now the extended lamellipodium needs to anchor to the extracellular matrix (ECM) via adhesion molecules such as integrins, a family of glycoproteins (Fig. 1b). ${ }^{9}$ Integrins are composed of $\alpha$ and $\beta$ subunits; the $\alpha v \beta 5$ integrin has a central
Dr Jose M Prieto read Pharmacy (1993) and obtained a PhD in Pharmacology (2001) at the University of Valencia (Valencia, Spain) in the field of topical inflammation. His Postdoctoral research activities include the EU funded projects 'Insect Chemical Ecology', under the late Prof. I. Morelli (Department of Bioorganic Chemistry, Universita degli Studi di Pisa, Italy) (2001-2004) and "Medicinal Cannabis" (Department of Pharmaceutical and Biological Chemistry, School of Pharmacy, University of London, United Kingdom) (2005-2006) with Prof. M. Heinrich. He was then appointed as Lecturer in Pharmacognosy (UCL School of Pharmacy) where his research focuses on elucidating the effects of medicinal plants and natural products on skin conditions (inflammation and cancer) using targeted lipidomics.

role in cellular migration. This can bind to several ECM proteins such as fibronectin, laminins and collagen, thereby promoting cell attachment and spreading. Various signalling pathways, such as active PI3K and protein kinase $\mathrm{C}$, induce the assembly of the focal complex in melanoma cells. ${ }^{6,12,13}$ All stages of melanoma are accompanied by the expression of $\alpha \mathrm{v}$, while $\beta 5$ is associated with the vertical growth phase and poor prognosis. $^{7}$

Also, there are other adhesion molecules that have the ability to mediate adhesion between two similar cells (homotypic adhesion) or two different ones (heterotypic interaction), and are essential for tumour cell intravasation and extravasation such as cadherins. ${ }^{7,10}$ Epithelial (E) and Neural (N) cadherins are the major subtypes of cadherin that are involved in keratinocyte-melanocyte adhesion. In healthy human skin, Ecadherin is expressed in melanocytes to ensure their attachment to keratinocytes. The intracellular domain of E-cadherin is fastened to the actin filaments at the cell edge via catenin, in order to resist any mechanical force that may detach a single cell from a tissue cell sheet. In malignant melanoma cells, there is a lack of expression of the E-cadherin and an overexpression of the $\mathrm{N}$-cadherin. The former causing the melanocytes to escape the regulatory mechanism of keratinocytes and the latter facilitating melanoma cell interactions with fibroblast and endothelial cells during invasion into distant tumour sites. ${ }^{5,11}$

\subsection{Secretion of surface protease and contraction}

For the cell to make its way through the three-dimensional matrix, surface proteases such as matrix metalloproteinases (MMPs) are recruited towards the attachment sites, hydrolysing nearby ECM substrates (Fig. 1c). ${ }^{6}$ Among 25 members of the MMP family, MMP-2, MMP-9 and MT1-MMP are upregulated in invasive melanoma cases. ${ }^{14}$ MMPs regulation depends on many nuclear transcription factors including activating protein1 (AP-1), nuclear factor-kappaB (NF- $\mathrm{BB})$, activating transcription factor-2 (ATF-2), cAMP response elementbinding (CREB), hypoxia-inducible factor (HIF), as well as a number of cell signaling proteins such as tumour necrosis factor- $\alpha$ (TNF- $\alpha)$, IL-6, IL-1 $\beta$, and granulocyte macrophage colony-stimulating factor (GM-CSF). ${ }^{15,} 16$ In addition, the Ras/PI3K/Akt signal pathway regulates cell metastasis through the activation of Akt, which eventually controls the expression 
of MMPs and governs many cellular processes implicated in tumorigenesis. ${ }^{17}$

MMPs are naturally found in a complex with their natural inhibitors, known as tissue inhibitors of metalloproteinases (TIMPs), but those are reduced in metastatic melanoma cell lines. ${ }^{18}$ Furthermore, MMPs are also involved in the vasculogenesis process by enhancing the angiogenic effects of pro-inflammatory cytokines e.g. interleukin-2 (IL-2); nitric oxide (NO) and vascular endothelial growth factor (VEGF). ${ }^{19}$

Once the cell is detached from the ECM substrate, apart from the focal points at either edge, contractile force is required to allow the bulk of the cell body to move forward, which depends on actomyosin contractility (Fig. 1c). Fibre assembly and contraction is promoted by RhoC through its binding to the downstream effector, the Rho-associated serine/threonine kinase (ROCK), controlling the actin dynamics in malignant melanoma cells. ${ }^{5,9}$

\subsection{Detachment}

Finally, the trailing edge of the cell detaches from the ECM by the concerted action of a number of proteins. Importantly, some dedicated kinases such as focal adhesion kinase (FAK) and extracellular signal-regulated kinase (ERK) are overexpressed in melanoma thus destabilizing focal complexes and facilitating cell migration (Fig. 1d). ${ }^{5,9}$ The cell is now free to repeat the cycle and keep moving forward eventually leaving the tumour primary site.

\section{Melanoma and anti-migratory natural products}

A number of plant derived natural products have been described as potential alternative therapies for metastatic cancers. ${ }^{20}$ The metastatic properties of melanoma cells are a major challenge to therapy. Therefore blocking the migratory and invasive capacity of malignant cells with phytochemicals may open the door to an effective strategy for melanoma treatment and/or prevention. ${ }^{21}$ We here review those natural single chemical entities and herbal extracts which have been specifically investigated for their direct in vivo and in vitro effects on melanoma migration. For those with identified intracellular mechanisms of action we provide their chemical structures and map their molecular activities (Fig. 2).

\subsection{Alkaloids}

\subsubsection{Berberine}

Berberine 1, an isoquinoline alkaloid, is extracted from the rhizome, roots and stem bark of a number of medicinal plants, mainly of the Berberis genus such as Berberis aquifolium Pursh. It is widely used in traditional medical systems such as the Ayurvedic and the Chinese systems. It possesses various pharmacological properties such as anti-diabetic and anticarcinogenic activity against various tumour cells, with insignificant cytotoxicity to normal cells. As tumour progression is associated with the overexpression of COX-2 and $\mathrm{PGE}_{2}$, the effect of berberine on both has been investigated. Singh et al. found that treatment of melanoma cells with berberine suppressed the invasion of the cells in a dose and time-dependent fashion and that this activity was correlated with the inhibition of COX-2, the expression of prostaglandin receptors EP2 and EP4, and the reduction in the level of $\mathrm{PGE}_{2}$.
Moreover, similar effects were detected in transfected cells with COX-2 small interfering RNA, confirming that COX-2 is a therapeutic target for berberine. In addition, this alkaloid was able to inhibit TPA- and $\mathrm{PGE}_{2}$-promoted cell migration in melanoma cells with reduced levels of NF- $\mathrm{BB}$, The antimigratory effect of berberine was not due to inhibition of cell viability or induction of cell apoptosis. 22 Interestingly, berberine is a generator of reactive oxygen species, and this leads to a reduction of the activity of ERK-1/2, ATF-2 and CREB. Moreover, berberine was able to inhibit lung metastasis in melanoma-bearing C57BL/6 mice models, demonstrating its anti-metastatic potential in melanoma cells. ${ }^{23,24}$

Berberine further suppresses the tumourgenicity of melanoma cells via its anti-angiogenic action in vitro and in vivo. It inhibited several pro-angiogenic factors, such as VEGF, inducible nitric oxide synthase (iNOS), and HIF, as well as proinflammatory mediators such as IL-1 $\beta$, IL-6, TNF- $\alpha$, and GMCSF. It also elevated anti-angiogenic factors like IL-2 and TIMP, leading to the suppression of tumour-directed capillary formation. ${ }^{19}$

\subsubsection{Harmine}

Harmine 2, is a naturally occurring beta-carboline alkaloid present in a number of medicinal plants such as Peganum harmala L. It is frequently used in folk medicine for the treatment of various diseases, as it is known for its neuropharmacological, anti-bacterial, anti-plasmodial and antitumour effects. In vitro and in vivo experiments conducted by Hamsa and Kuttan have shown the potential anti-migrative properties of harmine; it has inhibited tumour nodule formation in the lungs of melanoma-bearing C57BL/6 mice and reduced various biochemical parameters associated with lung metastasis. The reported activity was associated with the downregulation of the expression of pro-metastatic genes, such as MMP-9, ERK, and VEGFs, which play a major role in melanoma cell invasion. ${ }^{15}$ Another study reported that harmine displayed an anti-angiogenic effect, which was linked to the inhibition of tumour-directed capillary formation in vivo. A significant reduction of the level of serum pro-angiogenic factors such as VEGF and NO was detected in harmine-treated animals. Moreover, harmine deactivated NF- $\kappa \mathrm{B}-$ as well as other transcription factors like CREB and ATF-2- resulting in the inhibition of COX-2, iNOS and MMPs expression, all of which are involved in cancer progression. ${ }^{25}$<smiles>COc1ccc2cc3[n+](cc2c1OC)CCc1cc2c(cc1-3)OCO2</smiles><smiles>COc1ccc2c(c1)[nH]c1c(C)nccc12</smiles><smiles>O=C(/C=C/c1ccc(O)cc1)NCCc1c[nH]c2ccc(O)cc12</smiles> 


\subsubsection{Ipobscurine}

Ipobscurine $\mathbf{3}$ is an indole alkaloid extracted from Ipomoea obscura (L.) Ker Gawl., which exhibits anti-inflammatory and immunomodulatory activity. The migratory capacity of melanoma cells was reduced by ipobscurine treatment which was associated with a significant decrease in the production of MMPs and the expressions of VEGF, COX-2, and NO synthase. In addition, ipobscurine elevated TIMP-1 and IL-2, whereas the high levels of pro-inflammatory factors such as IL$1 \beta$, IL- 6 , TNF- $\alpha$, and GM-CSF were reduced in ipobscurinetreated animals. ${ }^{26}$

\subsection{4 $\quad \alpha$-Solanine}

$\alpha$-Solanine 4 is a glycoalkaloid that accumulates under certain conditions in sprouts and tubers of the Solanaceae species such as Solanum nigrum L. The results of in vitro studies demonstrated that non-toxic doses of $\alpha$-solanine suppressed the phosphorylation of $\mathrm{PI} 3 \mathrm{~K}$ and Akt without effecting the phosphorylation of ERK. The activity of MMP-2/9 and its regulator, NF- $\mathrm{BB}$, were inhibited when melanoma cells were exposed to this phytochemical. Taken together, the data revealed $\alpha$-solanine to be a new anti-metastatic agent. ${ }^{27}$

\subsubsection{0-Methoxy-9-nitrocamptothecin (MONCPT)}

MONCPT 5 is a semisynthetic derivative of camptothecin, an anticancer alkaloid isolated from Camptotheca acuminate Decne., which acts as a topoisomerase I inhibitor. These in vivo and in vitro results indicate that MONCPT decreased B16F10 pulmonary metastasis in tumour-bearing C57BL/6 mice and suppressed the cell invasion and migration without any cytotoxic effects. This was associated with a reduction in the secretion of MMP-9 and VEGF, and expression of HIF- $1 \alpha$. Moreover, it suppressed the phosphorylation level of ERK in B16F10 cells. ${ }^{28}$

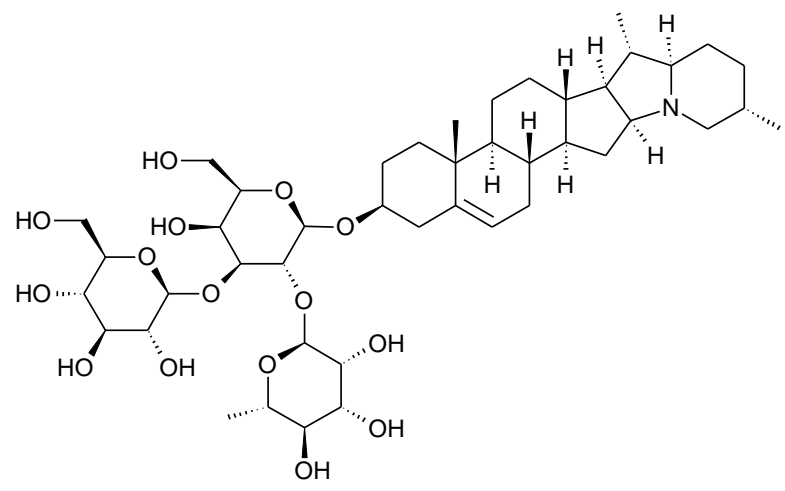

4

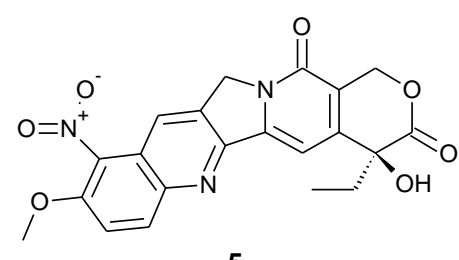

5

$$
\mathrm{C}_{12} \mathrm{H}_{22} \mathrm{~N}_{2} \mathrm{O}
$$

Structure not available

\subsubsection{Punarnavine}

Punarnavine 6, is a quinolizidine alkaloid of unknown structure isolated from Boerhaavia diffusa L. It exhibits analgesic, antiinflammatory, hepato-protective, immunomodulatory and antiproliferative properties. Its anti-metastatic properties were investigated using B16F-10 melanoma cells in C57BL/6 mice, and the administration of punarnavine significantly improved the survival rate of the metastatic tumour-bearing animals. This improvement was associated with the reduction in MMP-2, MMP-9 ERK-1/2 and VEGF in the lung tissue of metastasisinduced animals. ${ }^{29}$

\subsubsection{Other alkaloids}

The anti-migratory properties of other seven alkaloids have been reported in experimental melanoma models without full elucidation of their mode of action. Evodiamine is one of the main constituents of Evodia rutaecarpa (A. Juss.) Hook.f. \& Thomson and has been shown to inhibit melanoma cell invasion in vitro in a concentration manner. ${ }^{30}$ In addition, several anti-cancer agents were investigated for their effects on the migration process, including taxol (from Taxus brevifolia Nutt.), harringtonine and homoharringtonine (from Cephalotaxus hainanensis H.L.Li). These agents were found to be effective for the inhibition of cell invasion and migration of B16-BL6 cells. ${ }^{31}$ As taxol stabilizes microtubules, it causes cytoskeletal changes leading to a reduction in the metastatic properties of bladder cancer cells; ${ }^{32}$ however the impact of taxol on the cytoskeleton and metastatic properties of melanoma cells remains to be investigated. Moreover, sanguinarine, extracted from Sanguinaria canadensis L., reduced the tumour burden in melanoma-bearing C57BL/6 mice and in a human tumour xenograft grown in immunodeficient mice. ${ }^{21}$ Two other alkaloids, colchicine (from Colchicum autumnale L.) and cytochalasin B (from Curvularia lunata (Wakker) Boedijn.), were able to suppress tumour metastasis in C57/BL6 mice given IV injections of B16-F10 cells as well as to reduce the rate of migration from agarose droplets and the formation of homotypic aggregates. ${ }^{33}$

\subsection{Polyphenols}

\subsubsection{Genistein}

Genistein $\mathbf{7}$ is a constituent of Genista tinctoria L. which is endowed with several pharmacological properties such as antineoplastic and estrogenic activities. A recent report has indicated that genistein blocks the migration of melanoma cells without showing obvious cellular cytotoxicity at low doses. It seems to suppress early adhesion-induced protein tyrosine phosphorylation of focal complex proteins that results in an impairment of cell-ECM interaction. The result is a decreased invasive potential of B16-BL6 cells through the basement membrane. ${ }^{34}$ Moreover, it significantly reduced cell motility and experimental pulmonary metastatic foci in C57BL/6 mice, as well as tumour-induced angiogenesis. ${ }^{35,36}$

\subsubsection{Proanthocyanidins}

Proanthocyanidin $\mathbf{8}$ gives name to a large class of polyphenols. It have been reported to exhibit anti-carcinogenic effects in some tumour models. They exist in a variety of plant species such as Theobroma cacao L. They are usually present in complex mixtures made up of dimmers, trimers, tetramers, and 
oligomers of monomeric catechins and/or (-)-epicatechins. Due to this complexity, it is believed that their melanoma preventative effects is linked to the synergistic effects of some of these constituents, which in addition to being cytotoxic or antimigratory are able to act as sun blockers, thus preventing UV radiation-induced skin cancer in vivo. Vaid et al. have reported that treating melanoma cells with proanthocyanidins resulted in the concentration-dependent inhibition of cell migration, which was associated with the reduction in $\mathrm{PGE}_{2}$ production and down-regulation of the expression of COX-2 Proanthocyanidins significantly blocked the phosphorylation of ERK-1/2 and the promoted cell migration induced by TPA as the tumour promoter mediating CO-2 expression. In addition, proanthocyanidins were able to suppress NF- $\kappa \mathrm{B}$ activity linked with COX-2 regulation. Moreover, adhesion molecules involved in the process of epithelial transition in melanoma cells were upregulated, such as E-cadherin, and mesenchymal molecules (fibronectin and N-cadherin) were decreased due to proanthocyanidin treatment. ${ }^{37}$

\subsubsection{Anthocyanins}

The anthocyanins are present in the leaves, fruits and flowers of many highly pigmented plants and are reputed to possess antibacterial, antibiotic, anti-inflammatory, anti-oxidative, and immune-modulatory properties in folk medicine. Non-cytotoxic concentrations of a total extract of anthocyanins from Morus alba L. have been shown to inhibit the in vitro migratory capacity of melanoma cell in a Boyden chamber assay. Also, the expression of MMP-2/9, Ras, PI3K, Akt, and NF- $\kappa \mathrm{B}$ in C57BL/6 mice induced with B16-F1 cells were reduced, and this was accompanied by a reduction of pulmonary colonies in mice. Cyanidin-3-O- $\beta$-glycosides 9 are some of the most abundant anthocyanins in mulberries. ${ }^{17}$

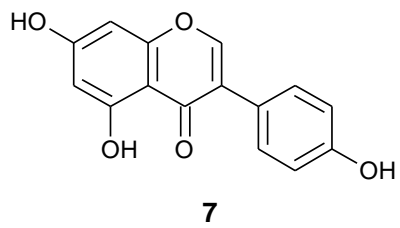<smiles>COc1c(O)cc(C2Oc3c(c(O)cc(O)c3C3c4c(O)cc(O)cc4O[C@H](c4ccc(O)cc4)[C@H]3O)C[C@H]2O)cc1O</smiles><smiles></smiles>

9

\subsubsection{Silymarin}

Silymarin $\mathbf{1 0}$ is a flavonoid complex isolated from milk thistle (Silybum marianum Gaertn.) and is composed up to $90 \%$ of silibinin 10a plus other isomers such as isosilybin 10b, silychristin 10c and silydianin 10d. There have been several reports that silymarin possesses anti-inflammatory, antioxidative and chemopreventative, and anti-carcinogenic properties. The topical application of silymarin has been shown to inhibit ultraviolet radiation-induced skin carcinogenesis in SKH-1 hairless mice and to reduce skin tumourgenesis, in a study showing that tumour incidence, tumour multiplicity and tumour growth were decreased. Vaid et al. studied the antimetastatic effects of silymarin in two special melanoma cell lines possessing constitutively active or inactive $\mathrm{Wnt} / \beta$-catenin signalling capabilities, respectively. As a result, they demonstrated that this flavonoid inhibits $\beta$-catenin translocation with subsequent down-regulation of MMPs expression. These observations confirm that $\beta$-catenin is the molecular target of silymarin. ${ }^{38}$<smiles>COc1cc(C2Oc3cc([C@@H]4Oc5cc(O)cc(O)c5C(=O)C4O)ccc3O[C@H]2CO)ccc1O</smiles>

$10 a$<smiles>COc1cc(C2Oc3ccc(C4Oc5cc(O)cc(O)c5C(=O)C(O)C4CO)cc3OC2CO)ccc1O</smiles>

$10 \mathrm{~b}$<smiles>COc1cc(C2Oc3c(O)cc(C4Oc5cc(O)cc(O)c5C(=O)[C@@H]4O)cc3C2CO)ccc1O</smiles>

$10 \mathrm{c}$

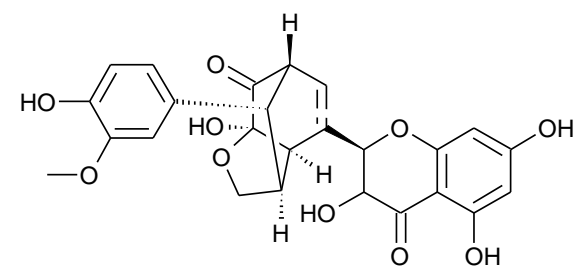

10d 


\subsubsection{Oroxylin A}

Oroxylin A 11, a flavonoid extracted from Scutellaria baicalensis Georgi, was able to suppress the expression and the activity of MMP-2/9, as detected by gelatin zymography, realtime PCR and western blotting analysis. The level of TIMP-2 was elevated and phosphorylation of ERK-1/2 and AP-1 was suppressed, which was associated with the inhibition of lung metastasis of B16-F10 cells in vivo. ${ }^{39}$

\subsubsection{Quercetin and apigenin}

Quercetin 12 and apigenin 13 have been very much researched for their anti-oxidant, anti-inflammatory and anti-cancer properties. They are found together in commonly used fruits and vegetables such as parsley (Petroselinum crispum (Mill.) Fuss). They significantly decreased lung colonization by B16BL6 in treated syngeneic C57BL/6N mice in a dose-dependent manner. ${ }^{40}$ Quercetin had anti-migratory effects against melanoma both alone and in combination with sulforaphane. The inhibitory effect of quercetin was due to a reduction of MMP-9 expression in the mouse tumours. ${ }^{41}$<smiles>COc1c(O)cc2oc(-c3ccccc3)cc(=O)c2c1O</smiles>

11<smiles>O=c1c(O)c(-c2ccc(O)c(O)c2)oc2cc(O)cc(O)c12</smiles>

12<smiles>O=c1cc(-c2ccc(O)cc2)oc2cc(O)cc(O)c12</smiles>

13

\subsubsection{Catechins}

Catechins 14 are a class of flavonoids best known for their prominence in tea leaves (Camellia sinensis (L.) Kuntze). They exhibit a number of physiological effects and health benefits, including antioxidant, anti-microbial and anti-carcinogenic activity against various cancer cells. Several catechins have been evaluated for their anti-migratory properties against melanoma cells, and the strength of their inhibitory effect is as follows: (-)-epigallocatechin-3-gallate (EGCG) 14a $>(-)$ epigallocatechin 14b $>(-)$-epicatechin-3-gallate 14c $>(-)$ gallocatechin 14d $>(-)$-epicatechin 14e. ${ }^{42}$<smiles>O=C(O[C@H]1Cc2c(O)cc(O)cc2O[C@@H]1c1cc(O)c(O)c(O)c1)c1cc(O)c(O)c(O)c1</smiles>

$14 a$<smiles>CS(=O)(=O)c1cc(O)c(O)c(O)c1</smiles><smiles>Oc1cc(O)c2c(c1)OC(c1cc(O)c(O)c(O)c1)[C@H](O)C2</smiles>

14d<smiles>Oc1cc(O)c2c(c1)O[C@H](c1ccc(O)c(O)c1)[C@H](O)C2</smiles>

$14 e$

EGCG -the most effective catechin- has been shown to inhibit melanoma cell migration in vitro and in vivo through multiple mechanisms and different molecular targets. The expression of COX-2 was diminished in melanoma cells after exposure to EGCG, resulting in the reduction of the levels of both $\mathrm{PGE}_{2}$ and its receptors, EP2 and EP4. This was associated with the inhibition of $\mathrm{NF}-\mathrm{\kappa B}$, the upstream regulator of $\mathrm{COX}-2$ expression that is implicated in the development of inflammation-induced cancer. ${ }^{42}$ In addition, EGCG was able to restore the level of epithelial adhesion molecules such as cadherin-E and to suppress the level of mesenchymal biomarkers such as fibronectin and $\mathrm{N}$-cadherin in melanoma cells. Wu and colleagues demonstrated the up-regulation of the expression of E-cadherin in a time and concentration manner. ${ }^{43}$ Interestingly, EGCG inhibits melanoma cellular migration without affecting cell viability or inducing cellular death, and no anti-migratory effect was observed in normal human epidermal melanocytes. These observations suggest that EGCG suppresses melanoma invasion via the reduction of the expression of COX-2 and $\mathrm{PGE}_{2}$ receptors and targeting of the melanoma cell interactions with the surrounded epithelial and mesenchymal cells. ${ }^{42}$ Liu and colleagues reported that, EGCG inhibited the activity of MMP-9, tyrosine phosphorylation of FAK and cell spreading on fibronectin, collagen, and matrigel. A significant reduction in the number of pulmonary metastases 
in Male Balb/c mice bearing B16-F3m melanoma was observed after EGCG treatment. Importantly, they reported on the synergistic effect of the combination of EGCG and dacarbazine in reducing lung metastasis in these mice, thereby increasing their survival rate, suggesting the clinical potential of EGCG in melanoma. ${ }^{44}$

Furthermore, the potential of EGCG in angiogenesis has been also investigated. It was found to suppress the phosphorylation of Akt in tumour-associated endothelial cells and VEGF. ${ }^{45}$ This was accompanied by the regulation of pro-inflammatory cytokines such as IL-1 $\beta$, IL-6, TNF- $\alpha$, and GM-CSF. Furthermore, EGCG elevated the level of anti-tumour factors (IL-2 and TIMP-1) and decreased the level of angiogenesis promoters (NO and VEGF) as well as mRNA levels of VEGF in B16F-10 melanoma cells. ${ }^{46}$

\subsubsection{Curcumin}

Curcumin 15, is a pigmented product obtained from the rhizome of Curcuma longa L., which is traditionally used to treat inflammatory diseases. Some authors have demonstrated its inflammatory, anti-viral and anti-tumour properties.<smiles>COc1cc(/C=C/C(=O)CC(=O)/C=C/c2ccc(O)c(OC)c2)ccc1O</smiles>

15

Its anti-invasive properties have been investigated with regard to melanoma cells by Menon et al. They found that curcumin downregulated the expression of MMPs and NF- $\mathrm{BB}$-mediated MMP-2 activation, and also inhibited the expression of MMPs in osteopontin-induced cells migration. ${ }^{47}$ The in vivo inhibitory effect of curcumin was demonstrated through the suppression of lung metastasis induced by B16-F10 melanoma cells in C57BL/6 mice. ${ }^{48}$ Also, curcumin has been shown to inhibit the phosphorylation of Src kinase and STAT3 pathway through the down-regulation of phosphatase of regenerating liver-3 (PRL3 ), which plays a role in cancer metastasis. ${ }^{49}$

\subsubsection{Amentoflavone}

Amentoflavone $\mathbf{1 6}$ is a bioflavonoid found in various plants including Ginkgo biloba L. and Hypericum perforatum L. Several in vitro properties of amentoflavone have been reported, such as anti-malarial and anti-cancer activities. A study by Tarallo et al. showed that amentoflavone blocked the interaction and phosphorylation of VEGF with its receptors, VEGF receptors 1 and 2 (VEGFR-1/-2). Moreover, it inhibited endothelial cell migration and capillary-like tube formation. ${ }^{50}$ In vivo experiments revealed that it decreased the level of various endogenous factors such as IL- $1 \beta$, IL- 6 and GM-CSF and it elevated the level of IL-2 and TIMP-1, confirming that amentoflavone acts as an antiangiogenic molecule. In addition, it reduced experimental tumour metastasis in syngeneic C57BL/6 mice implanted with B16 cells. Guruvayoorappan and Kuttan demonstrated that amentoflavone was able to reduce the mRNA expression of MMP-2/9, ERK-1/2 and TNF- $\alpha$ in lung tissues while the expression of STAT-1 and nm23, which play a key role in the acquisition of melanoma metastatic ability, was increased. ${ }^{51,52}$

\subsubsection{Capsaicin}

Capsaicin $\mathbf{1 7}$ is the main active compound in the chili pepper (Capsicum frutescens L.), one of the most commonly used spices in the world, and other plants of the Capsicum genus. Analgesic, anti-inflammatory and anti-cancer activities of capsaicin through the induction of apoptosis have been reported. The anti-migratory activity of capsaicin on melanoma cells was correlated with inhibition of the PI3K/Akt/Rac1 signal pathway. The observed activity was associated with the down-regulation of PI3K and its downstream target, Akt, as well as the inhibition of Rac1 activity. ${ }^{53}$ The potential of capsaicin to inhibit melanoma invasion has been investigated by Min et al. and it has been related to the inhibition of VEGF activity and capillary-like tube formation of primary cultured human endothelial cells. ${ }^{43}$<smiles>O=c1cc(-c2ccc(O)c(-c3c(O)cc(O)c4c(=O)cc(-c5ccc(O)cc5)oc34)c2)oc2cc(O)cc(O)c12</smiles>

16<smiles>COc1cc(CNC(=O)CCCC/C=C/C(C)C)ccc1O</smiles>

\subsubsection{Resveratrol and pterostilbene}

Resveratrol 18a is a natural phenol which possesses antioxidant and inflammatory properties, found in Polygonum cuspidatum Willd. ex Spreng and red wine. Its anti-migratory properties against melanoma cells have been shown in vitro and in vivo. These effects seem to be related to the deactivation of Akt, as determined by a combination of real-time PCR and western blot analysis. ${ }^{54}$ However, the activity of resveratrol is limited by its low bioavailability. Pterostilbene 19, which is a related natural polyphenol existing in a high concentration in berries and grapes such as Vitis vinifera L., shows similar activity to resveratrol but with the advantage of a longer half-life in vivo. In fact, half-life of pterostilbene after I.V. administration to mice was five fold longer $\left(\mathrm{t}_{1 / 2}=77.9 \mathrm{~min}\right)$ than this of resveratrol $\left(t_{1 / 2}=14.4\right.$ min $) .{ }^{55}$ Furthermore, the I.V. administration of pterostilbene in C57BL/6J mice inhibits the metastatic growth of melanoma cells to the liver. The antimetastatic mechanism involves the inhibition of the expression of vascular adhesion molecule 1 , which plays a central role in the migration process, leading to a reduction in the rate of adhesion between melanoma cells and the vascular endothelium. ${ }^{56}$ 
The enormous potential of resveratrol led to the synthesis of derivatives to enhance its pharmacological activities; one of these is the stilbene $3,3^{\prime}, 4,4^{\prime}, 5,5^{\prime}$-hexahydroxy derivative $\mathbf{1 8 b}$. It has been shown to decrease the migratory properties of melanoma cells in vitro through interference with integrinlinked protein kinase and ROCK-1, indicating its role in the inhibition of mesenchymal and amoeboid cell migration. ${ }^{57}$<smiles>[R6]c1cc(/C=C/c2cc([R6])c([R6])c(Br)c2)cc([R6])c1[R5]</smiles>

18a $\mathrm{R}_{1}, \mathrm{R}_{2}, \mathrm{R}_{3}, \mathrm{R}_{5}=\mathrm{H} \quad \mathrm{R}_{4}, \mathrm{R}_{6}=\mathrm{OH}$

$18 \mathrm{~b} \mathrm{R} \mathrm{R}_{1}, \mathrm{R}_{2}, \mathrm{R}_{3}, \mathrm{R}_{4}, \mathrm{R}_{5}, \mathrm{R}_{6}=\mathrm{OH}$

$19 \mathrm{R}_{1}, \mathrm{R}_{3}, \mathrm{R}_{5}=\mathrm{H} \quad \mathrm{R}_{2}=\mathrm{OH} \quad \mathrm{R}_{4,6}=\mathrm{OCH}_{3}$

\subsubsection{Gallic acid}

Gallic acid 20 is a common plant metabolite known to possess antibacterial, antifungal, antimalarial, antioxidant and antiviral action. The inhibitory potential of this simple phenol against the invasion of melanoma cells is mediated, at least in part, through the Ras/ERK signalling pathways leading to the reduction of MMP-2/9 gene expression and protein levels. ${ }^{58}$

\subsubsection{Gambogic acid}

Gambogic acid $\mathbf{2 1}$ is a haemostatic and an antiseptic xanthonoid isolated from the resin of Garcinia hanburyi Hook.f. It has been reported to inhibit the metastatic potential of the B16-F10 melanoma cell line in vitro and in vivo. The down-regulation of $\alpha 4$ integrin expression in association with negligible effects on the expression of $\alpha 5$ and $\beta 1$ integrin was involved in this anti-metastatic action, as detected by flowcytometry and western blot analyses. ${ }^{59}$

\subsubsection{Other flavonoids}

The effects of two other flavonoids on the invasion and metastasis of B16 cells have been reported without description of the mechanisms of action. Ampelopsin, derived from Cedrus deodara (Roxb. ex D.Don) G.Don, reduced the in vitro ability of these melanoma cells to migrate and this observation correlated with a reduction in the number of metastases in treated $\mathrm{C} 57 \mathrm{BL} / 6$ mice. $^{60}$ In addition, a quercetin derivative extracted from Cladocolea micrantha (Eichler) Kuijt, bearing an $O$-arabinofuranosyl- $(1 \rightarrow 3)$-rhamnoside moiety, promoted a twofold inhibition of melanoma cell migration in vitro. ${ }^{61}$<smiles>O=C(O)c1cc(O)c(O)c(O)c1</smiles>

20

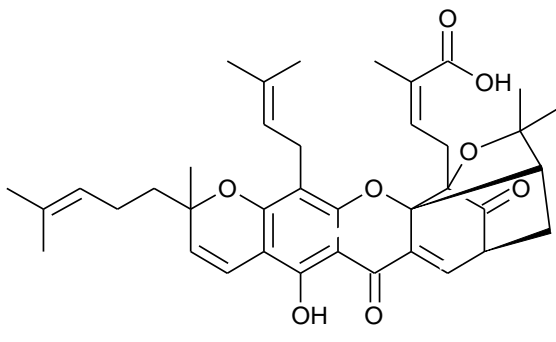

21

\subsection{Vitamin-related compounds}

\subsubsection{Retinoic acid}

Retinoic acid 22 is an all-trans metabolite of vitamin A that mediates gene transcription and regulates a wide variety of normal cellular processes such as cell proliferation and differentiation. Retinoic acid exhibits a pronounced antiinvasive effect and appears to inhibit the adhesion of melanoma cells to the extracellular matrix components type I collagen and laminin. Using fluorescence microscopy, Helige et al. have demonstrated that it can modulate the organization of the actin cytoskeleton. ${ }^{62}$ Also, retinoic acid reduced the level of collagenolytic enzymes and type IV collagenase messenger RNA and decreased the activity of tissue plasminogen activator. Moreover, retinoic acid treatment was associated with a reduction in the expression of cell-surface receptors for motility factors. ${ }^{63}$ In addition, retinoic acid was able to block the neovascularization process by elevating the production of IL-2 and TIMP-1 in treated animals and blocking the activation of NF- $\mathrm{BB}$ subunits, p65, p50, c-Rel, and other transcription factors such as c-fos, ATF-2 and CREB protein in melanoma cells. ${ }^{64}$<smiles>CC1=C(/C=C/C(C)=C/C=C/C(C)=C/C(=O)O)C(C)(C)CCC1</smiles>

\subsubsection{Riboflavin photoproducts}

Irradiation of Vitamin B2 with UV light leads to the formation of a complex mixture of photolytic products (iRFs) endowed with interesting biological activities. Among those, they disrupt melanoma cell migration, increase TIMP expression and suppress the activity and expression of MMP in treated cells. This is due , at least in part, by targeting the hedgehog pathway, which regulates the metastasis of melanoma. iRFs upregulate SUFU, an inhibitor of this pathway, and downregulate GLI1 and $\mathrm{PTCH}$. In addition, they reduce the incidence of pulmonary metastases in treated $\mathrm{C} 57 \mathrm{Bl} / 6$ mice. ${ }^{65}$

\subsection{Terpenoids}

\subsubsection{Artemisinin}

Artemisinin $\mathbf{2 3}$ is a known anti-malarial agent; it is a sesquiterpene derived from Artemisia annua L., which is used in traditional Chinese medicine as an antipyretic. It has been shown to target the adhesion capacity of melanoma cells via the down-regulation of $\alpha 5 \beta 3$ integrin expression, as well as reducing MMP-2 production, revealing the potential chemotherapeutic effect of artemisinin in melanoma treatment. 66

\subsubsection{Carnosol, Atractylenolides I and II}

Carnosol 24, a component of Rosmarinus officinalis L., as well as atractylenolides I $\mathbf{2 5}$ and II 26, from Atractylodes macrocephala Koidz, have shown potent in vitro activity against melanoma migration. All of the compounds inactivated ERK phosphorylation and inhibited Akt, suggesting that the PI3K/Akt signalling pathway is involved in their action. ${ }^{67,68}$ 
Carnosol inhibited MMP activity as well as NF- $\mathrm{BB}$ and AP-1 binding activity, thereby restricting the invasive ability of melanoma cells. ${ }^{68}$

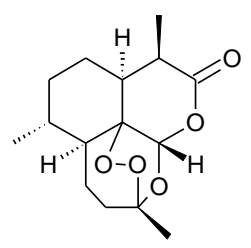

23<smiles>C=C1CCCC2(C)CC3OC(=O)C(C)=C3CC12</smiles>

25

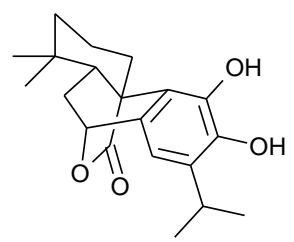

24

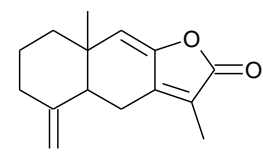

26

\subsubsection{Thujone and Vernolide-A}

Thujone $\mathbf{2 7}$ is a monoterpene found in a number of plants, e.g. Thuja standishii Gordon, and vernolide-A 28 is a sesquiterpene lactone present in Vernonia cinerea L. Their anti-metastasis properties have been reported, where the administration of both compounds significantly suppressed tumour nodule formation in the lungs of C57BL/6 mice transplanted with B16 melanoma cells. These effects were accompanied by the down-regulation of the expression of MMP-2/9, ERK-1/2, and VEGF in the lung tissue of treated animals. ${ }^{69,70}$ In addition, thujone treatment reduced the production of pro-inflammatory factors such as TNF- $\alpha$, IL-1 $\beta$, IL-6, and GM-CSF, whereas the expression of TIMP-1/2 was elevated. ${ }^{69}$

\subsubsection{Parthenolide}

The sesquiterpene lactone parthenolide $\mathbf{2 9}$ is the putatitve active principle of Tanacetum parthenium (L.) Sch.Bip. (Feverfew), a herbal medicine used to treat several conditions, including inflammation, migraine, menstrual irregularities, fever and rheumatoid arthritis. Parthenolide has been found to be a potential agent against melanoma migration through its inhibitory effect on the constitutive and induced NF- $\mathrm{BB}$ pathway. It also suppressed IL-8 and MMP-9 expression, which are involved in melanoma migration and invasiveness. ${ }^{71}$

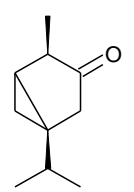

27

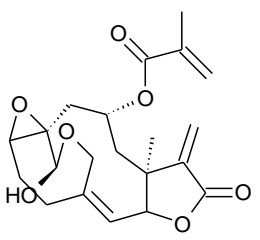

28

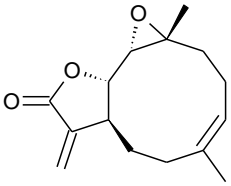

29

\subsection{5 $\quad \beta$-Carotene}

Carotenoids are tetraterpenes with multiple biological and pharmacological activities; $\beta$-carotene $\mathbf{3 0}$ is a carotenoid with photosynthetic and vitamin A activity present in many edible fruits and vegetables such as Daucus carota L. The inhibitory effect of $\beta$-carotene on melanoma metastasis was associated with the prevention of the activation of several subunits of NF$\kappa \mathrm{B} ; \mathrm{p} 65$, p50, and c-Rel. It alos elevated the expression level of TIMP-1/2 and down-regulated the expression of MMP-2/9, prolyl hydroxylase, as well as the lysyl oxidase gene involved in the metastasis process. ${ }^{72}$<smiles>CC1=C(/C=C/C(C)=C/C=C/C(C)=C/C=C/C=C(C)/C=C/C=C(C)/C=C/C2=C(C)CCCC2(C)C)C(C)(C)CCC1</smiles>

\subsubsection{Other terpenoids}

Oridonin, a diterpenoid isolated from Rabdosia rubescens (Hemsl.) H.Hara, has been shown to inhibit the motility of melanoma cells in a concentration manner. ${ }^{73}$ The treatment of C57BL/6 mice induced with metastatic B16F-10 melanoma cells with hypoestoxide (from Hypoestes rosea Decne.) and ursolic acid (from Ocimum sanctum L.) resulted in a marked reduction of lung metastatic colonies. The levels of serum VEGF and NO were decreased in ursolic acid-treated animals. $73,74,75$

\subsection{Peptides}

\subsubsection{Actibind}

Actibind is a T2 RNase extracted and purified from the fungus Aspergillus niger van Tieghem. As such, it degrades RNA thereby inhibiting protein synthesis and subsequent cell death. Actibind is effective as an anti-metastatic agent since it inhibited human melanoma cell invasion in vivo and in vitro by suppressing the activity of MMP-2 in a dose-dependent manner as well as inhibiting pro-MMP-2 collagenase activity in melanoma cells. ${ }^{76}$

\subsubsection{Glycoproteins}

Gintonin, a novel glycolipoprotein from Panax ginseng C.A.Mey., suppressed pulmonary metastasis induced by tailvein inoculations of melanoma cells in C57BL/6 mice. Its effect in cell migration contrats with minimal inhibition of tumour growth and is mediated by its antagonism to lysophosphatidic acid receptors. ${ }^{77}$ The related iminosugars were also able to inhibit pulmonary metastasis of the melanoma cells in treated mice, e.g. gem-diamine 1-N-iminosugars related to D-glucuronic acid and L-iduronic acid, nojirimycin and Dglucaro-d-lactam. ${ }^{78}$

Peptides from animal origin have been also investigated. Hirudin from leeches (Hirudo medicinalis L.) alone or in combination with liposomal vinblastine has exhibited a potent effect against tumour growth and metastasis of melanoma in vivo as well as melanoma adhesion in vitro. ${ }^{79}$ The Mohave rattlesnake (Crotalus scutulatus Kennicott) venom yields RMojastin 1 that suppressed SK-MEL-28 cell adhesion to fibronectin. Furthermore, it inhibited lung tumour colonization of melanoma cells in BALB/c mice. ${ }^{80}$ 


\subsection{Herbal extracts}

\subsubsection{Prunella vulgaris}

The in vitro and in vivo effects of an aqueous extract from Prunella vulgaris L. (PVAE) have been studied. MMP-9 expression and its mRNA level were diminished via the inhibition of the activity of $\mathrm{NF}-\kappa \mathrm{B}$ as well as blocking the phosphorylation of ERK-1/2. PVAE was able to suppress melanoma metastasis in tumour-bearing C57BL/6 mice. ${ }^{81}$ Its chemical composition includes alkaloids, saponins, phenolics and tannins. ${ }^{82}$

\subsubsection{Piper longum}

The anti-metastatic properties of an ethanolic extract of Piper longum $\mathrm{L}$. have been investigated in melanoma-bearing C57BL/6 mice. The level of cytokines involved in the metastasis process, such as IL-1 $\beta$, IL-6, VEGF, TNF- $\alpha$ and GM-CSF, was decreased. On the other hand, IL-2 and TIMP-1 levels were elevated, reducing the metastatic capacity of melanoma cells. ${ }^{83}$ The phytochemical analysis reveals that the herb contains alkaloids such as piperine, lignans and volatile oils. ${ }^{84}$ Piperine might contribute to the activity of the extract as it is a known anti-cancer lead compound, with inhibitory activity in the lung metastasis induced by B16F-10 melanoma cells in C57BL/6 mice. ${ }^{85}$

\section{FIGURE 2 MAY GO HERE (SEE AT THE END OF THE DOCUMENT)}

\section{Concluding remarks}

Natural products have emerged as a very promising source of anti-migratory agents, some of them being considered as potential treatment interventions. This review summarizes the experimental evidence of the anti-metastatic activity of natural products in melanoma and charts the mechanisms of action underlying these interesting properties (Fig. 2). Their most important intracellular targets appear to be the downstream inactivation of a number of nuclear factors, namely $\mathrm{NF}-\kappa \mathrm{B}$, AP1, ATF-2, CREB, and HIF. These effects are mediated, at least in part, by the inhibition of important kinases including PI3K, RAS, ERK and FAK. Antagonistic effects upon the TNF- $\alpha$ and EP2 receptors and the function of adhesion molecules -including integrin and $\mathrm{N}$-cadherin- were also described for a number of them. A majority of the reviewed products seem to be selectively acting upon one of these targets only, but remarkably some (berberine, harmine, EGCG and retinoic acid) are multitargeting several of these key pathways holding promise in future clinical studies.

These compounds could be clinically used on their own or in combination with another natural product or drug. The first option is only commercially viable when using patentable products. This can be achieved by modifying the original natural product such as in the case of colchicine and MONCPT 28 . The second one could make use of the natural product as such together with an approved drug, for example EGCG in combination with dacarbazine, which resulted in an improvement in the survival rate of melanoma-bearing mice. ${ }^{44}$ This also applies to the combination of two natural compounds such as in the case of quercetin and sulforaphane. 41 Interestingly, many of the molecules reviewed here could be obtained as by-products of the food industry. In some cases, nutritional interventions with standardized extracts enriched in the active natural product/s could be devised for chemoprevention in healthy individuals or improvement of cancer prognosis. In addition, some of the compounds discussed here are already used for clinical purposes for other indications in the form of derivatives such as artesunate, which may accelerate their use for melanoma treatment. In summary, we here highlight that many natural-derived agents have demonstrated their potential in the treatment/prevention of migration and how they could be incorporated into melanoma therapy.

\section{References}

1. D. L. Morton, R. Essner, J. M. Kirkwood and R. C. Wollman, Malignant Melanoma in Cancer Medicine, 2006.

2. S. Kuphal and A. Bosserhoff, J. Pathol., 2009, 219, 400-409.

3. J. Villanueva and M. Herlyn, Melanoma, eLS, John Wiley \& Sons Ltd, 2009.

S. Sharma and S. Katiyar, Pharmacol. Res., 2010, 27, 1092-1102. C. Gaggioli and E. Sahai, Pigment Cell Res., 2007, 20, 161-172.

P. Friedl and K. Wolf, Nat. Rev. Cancer, 2003, 3, 362-374.

E. C. McGary, D. C. Lev and M. Bar-Eli, Cancer Biol. Ther., 2002, 1, 459-465.

8. A. Howe, A. E. Aplin, S. K. Alahari and R. L. Juliano, Curr. Opin. Cell Biol., 1998, 10, 220-231.

9. M. Parri and P. Chiarugi, Cell Commun. Signal., 2010, 8, 23.

10. N. K. Haass, K. S. M. Smalley, L. Li and M. Herlyn, Pigment Cell Melanoma Res., 2005, 18, 150-159.

11. S. Kuphal and A. K. Bosserhoff, Arch. Biochem. Biophys., 2012, 524, 43-47.

12. P. Pinon and B. Wehrle-Haller, Pigment Cell Melanoma Res., 2011, 24, 282-294.

13. J. D. Hood and D. Cheresh, Nat. Rev. Cancer, 2002, 2, 91-100.

14. K. Shaverdashvili, P. Wong, J. Ma, K. Zhang, I. Osman and B. Bedogni, Pigment Cell Melanoma Res., 2013, n/a-n/a.

15. T. Hamsa and G. Kuttan, J. Environ. Pathol. Toxicol. Oncol., 2011, 30, 123-137.

16. J. H. Choi, E. H. Han, Y. P. Hwang, J. M. Choi, C. Y. Choi, Y. C. Chung, J. K. Seo and H. G. Jeong, Food Chem. Toxicol., 2010, 48, 564-571.

17. H. P. Huang, Y. W. Shih, Y. C. Chang, C. N. Hung and C. J. Wang, J. Agric. Food Chem., 2008, 56, 9286-9293.

18. P. Valente, G. Fassina, A. Melchiori, L. Masiello, M. Cilli, A Vacca, M. Onisto, L. Santi, W. G. Stetler-Stevenson and A. Albini, Int. J. Cancer, 1998, 75, 246-253.

19. T. P. Hamsa and G. Kuttan, Drug Chem. Toxicol., 2012, 35, $57-$ 70.

20. M. Lahlou, Pharmacol. Pharm, 2013, 4, 17-31.

21. I. De Stefano, G. Raspaglio, G. F. Zannoni, D. Travaglia, M. G. Prisco, M. Mosca, C. Ferlini, G. Scambia and D. Gallo, Biochem. Pharmacol., 2009, 78, 1374-1381.

22. T. Singh, M. Vaid, N. Katiyar, S. Sharma and S. K. Katiyar, Carcinogenesis, 2011, 32, 86-92.

23. T. P. Hamsa and G. Kuttan, Phytotherapy Res., 2012, 26, 568578.

24. H. S. Kim, M. J. Kim, E. J. Kim, Y. Yang, M. S. Lee and J. S. Lim, Biochem. Pharmacol., 2012, 83, 385-394.

25. T. P. Hamsa and G. Kuttan, Eur. J. Pharmacol., 2010, 649, 6473.

26. T. Hamsa and G. Kuttan, J. Environ. Pathol. Toxicol. Oncol., 2011, 30, 163-178.

27. M. K. Lu, Y. W. Shih, T. T. Chang Chien, L. H. Fang, H. C. Huang and P. S. Chen, Biol. Pharm. Bull., 2010, 33, 1685-1691.

28. X. C. Yang, C. X. Tu, P. H. Luo, H. Zhu, D. F. Zhu, H. H. Wu, X. L. Zhou, W. Lu, Q. J. He and B. Yang, Invest New Drugs, 2010, 28, 800-811.

29. K. A. Manu and G. Kuttan, Immunobiology, 2009, 214, 245-255.

30. M. Ogasawara, T. Matsunaga, S. Takahashi, I. Saiki and H. Suzuki, Biol. Pharm. Bull., 2002, 25, 1491-1493.

31. H. Liu, X. Lei and R. Han, Yao Xue Xue Bao,= Acta pharmaceutica Sinica, 1998, 33, 18-21. 
K. Inoue, J. W. Slaton, D. W. Davis, D. J. Hicklin, D. J. McConkey, T. Karashima, R. Radinsky and C. P. N. Dinney, Clin. Cancer Res., 2000, 6, 2635-2643.

33.

. 891-900. C. Yan and R. Han, Cancer Lett., 1998, 129, 117-124.

35. Y. Li, S. Upadhyay, M. Bhuiyan and F. Sarkar, Oncogene, 1999 18, $3166-3172$.

H. G. Farina, M. Pomies, D. F. Alonso and D. E. Gomez, Oncol. Rep., 2006, 16, 885-891. M. Vaid, R. Prasad, Q. Sun and S. K. Katiyar, PloS one, 2011, 6, e23000.

Z. Lu, N. Lu, C. Li, F. Li, K. Zhao, B. Lin and Q. Guo, Toxicol. Lett., 2012, 209, 211-220.

40. S. Caltagirone, C. Rossi, A. Poggi, F. O. Ranelletti, P. G. Natali, M. Brunetti, F. B. Aiello and M. Piantelli, Int. J. Cancer, 2000, 87, 595-600

41. S. J. Pradhan, R. Mishra, P. Sharma and G. C. Kundu, Exp. Ther. Med., 2010, 1, 915-920.

42. T. Singh and S. K. Katiyar, PLoS One, 2011, 6, e25224.

43. Y. Wu, Y. Lin, H. Liu and J. Li, J J. Huazhong Univ. Sci. Technol. Med. Sci., 2008, 28, 356-359.

$44 . \quad$ J. D. Liu, S. H. Chen, C. L. Lin, S. H. Tsai and Y. C. Liang, J. Cell. Biochem., 2001, 83, 631-642.

45. N. Ohga, K. Hida, Y. Hida, C. Muraki, K. Tsuchiya, K. Matsuda, Y. Ohiro, Y. Totsuka and M. Shindoh, Cancer Sci., 2009, 100, 1963-1970.

46.

Guruvayoorappan and G. Kuttan, Innate Immun., 2008, 14, 160-174.

47. S. Philip and G. C. Kundu, T J. Biol. Chem., 2003, 278, $14487-$ 14497.

48. L. G. Menon, R. Kuttan and G. Kuttan, Cancer Lett., 1999, 141, $159-165$.

49.

L. Wang, Y. Shen, R. Song, Y. Sun, J. Xu and Q. Xu, Mol. Pharmacol., 2009, 76, 1238-1245.

V. Tarallo, L. Lepore, M. Marcellini, F. Dal Piaz, L. Tudisco, S. Ponticelli, F. W. Lund, P. Roepstorff, A. Orlandi, C. Pisano, N. De Tommasi and S. De Falco, J. Biol. Chem., 2011, 286, 1964119651

51. C. Guruvayoorappan and G. Kuttan, Immunopharm. Immunot., 2008, 30, 711-727.

52. C. Guruvayoorappan and G. Kuttan, Biochemistry, 2008, 73, 209218.

53.

D. H. Shin, O. H. Kim, H. S. Jun and M. K. Kang, Exp. Mol. Med., 2008, 40, 486-494.

54. S. Bhattacharya, S. R. Darjatmoko and A. S. Polans, Melanoma Res., 2011, 21.

55. M. Asensi, I. Medina, A. Ortega, J. Carretero, M. C. Baño, E. Obrador and J. M. Estrela, Free Radic. Biol., 2002, 33, 387-398. P. Ferrer, M. Asensi, M. Segarra, A. Ortega, Mar\&iacute, A. Benlloch, E. Obrador, Mar\&iacute, A. V. Varea, G. Asensio, J. Leonardo and J. M. Estrela, Neoplasia, 2005, 7.

57. V. Paulitschke, N. Schicher, T. Szekeres, W. Jager, L. Elbling, A B. Riemer, O. Scheiner, G. Trimurtulu, S. Venkateswarlu, M Mikula, A. Swoboda, E. Fiebiger, C. Gerner, H. Pehamberger and R. Kunstfeld, J. Invest. Dermatol., 2010, 130, 1668-1679.

58. C. Lo, T. Y. Lai, J. S. Yang, J. H. Yang, Y. S. Ma, S. W. Weng, H. Y. Lin, H. Y. Chen, J. G. Lin and J. G. Chung, Melanoma Res. 2011, 21, 267-273.

59. J. Zhao, Q. Qi, Y. Yang, H.-Y. Gu, N. Lu, W. Liu, W. Wang, L. Qiang, L.-B. Zhang, Q.-D. You and Q.-L. Guo, Eur. J. Pharmacol., 2008, 589, 127-131.

60. D. Y. Liu, H. Q. Zheng and G. Q. Luo, Zhongguo Zhong Yao Za Zhi = China journal of Chinese materia medica, 2003, 28, 957 961.

61. A. C. Guimaraes, A. Magalhaes, M. J. Nakamura, A. C. Siani, C. Barja-Fidalgo and A. L. Sampaio, Nat. Prod. Commun., 2012, 7, 1311-1314.

62. C. Helige, J. Smolle, G. Zellnig, E. Hartmann, R. Fink-Puches, H Kerl and H. A. Tritthart, Clin. Exp. Metastasis, 1993, 11, 409-418. J. P. Ortonne, J. Dermatol., 1992, 127 Suppl 41, 43-47.

64. C. Guruvayoorappan and G. Kuttan, J. Exp. Ther. Oncol., 2008, 7, 173-182.

65.
66. E. Buommino, A. Baroni, N. Canozo, M. Petrazzuolo, R. Nicoletti, A. Vozza and M. A. Tufano, Invest. New Drugs, 2009 27, 412-418.

67. Y. Yan, G. X. Chou, W. Hui, J. H. Chu, W. F. Fong and Z. L. Yu, Integr. Cancer. Ther., 2011, 10, 92-100.

68. S. C. Huang, C. T. Ho, S. Y. Lin-Shiau and J. K. Lin, Biochem. Pharm, 2005, 69, 221-232.

69. K. S. Siveen and G. Kuttan, J. Physiol. Pharmacol., 2011, 89, 691-703.

70. P. Pratheeshkumar and G. Kuttan, Hum. and Exp. Toxicol., 2012, 31, 66-80.

71. M. Czyz, K. Lesiak-Mieczkowska, K. Koprowska, A. SzulawskaMroczek and M. Wozniak, Integr. Cancer. Ther., 2010, 160, 1144-1157.

72. C. Guruvayoorappan and G. Kuttan, Integr. Cancer. Ther., 2007, 6, 258-270.

73. K.-K. Ren, H.-Z. Wang, L.-P. Xie, D.-W. Chen, X. Liu, J. Sun, Y.-c. Nie and R.-Q. Zhang, J. Ethnopharmacol., 2006, 103, 176180.

74. E. A. Ojo-Amaize, E. J. Nchekwube, H. B. Cottam, R. Bai, P. Verdier-Pinard, V. N. Kakkanaiah, J. A. Varner, L. Leoni, J. I. Okogun, A. A. Adesomoju, O. A. Oyemade and E. Hamel, Cancer Res., 2002, 62, 4007-4014.

75. M. Kanjoormana and G. Kuttan, Integr. Cancer. Ther., 2010, 9 224-235.

76. B. Schwartz, O. Shoseyov, V. O. Melnikova, M. McCarty, M Leslie, L. Roiz, P. Smirnoff, G.-f. Hu, D. Lev and M. Bar-Eli, Cancer Res., 2007, 67, 5258-5266.

Hwang, Lee, Kim, Cho, Shin, Im, Choi, Shin, Lee, Nam, Kim, Rhim and Nah, Int. J. Oncol., 2013, 42, 317-326.

Y. Nishimura, Curr. Top. Med. Chem., 2003, 3, 575-591.

79. R. R. Guo, Y. Liu, W. L. Lu, J. H. Zhao, X. Q. Wang, H. Zhang, J. C. Wang, X. Zhang and Q. Zhang, Biol. Pharm. Bull., 2008, 31, 696-702.

80. S. Lucena, E. E. Sanchez and J. C. Perez, Toxicon, 2011, 57, 794 802.

81. J. H. Choi, E. H. Han, Y. P. Hwang, J. M. Choi, C. Y. Choi, Y. C. Chung, J. K. Seo and H. G. Jeong, Food Chem. Toxicol., 2010 48, 564-571.

82. A. Rafia, B. A. Ganai, S. Akbar, A. N. Kamili and A. Masood, Pak. J Pharm. Sci., 2010, 23, 399-402.

83. E. S. Sunila and G. Kuttan, Int. Immunopharmacol., 2006, 6, 733741.

84. C. Khushbu, S. Roshni, P. Anar, M. Carol and P. Mayuree, Int. J. Res. Ayurveda Pharm., 2011, 2, 157-161.

85. C. R. Pradeep and G. Kuttan, Clin. Exp. Metastas., 2002, 19, 703 708. 
Figure 1 The migration process in cancer cells.

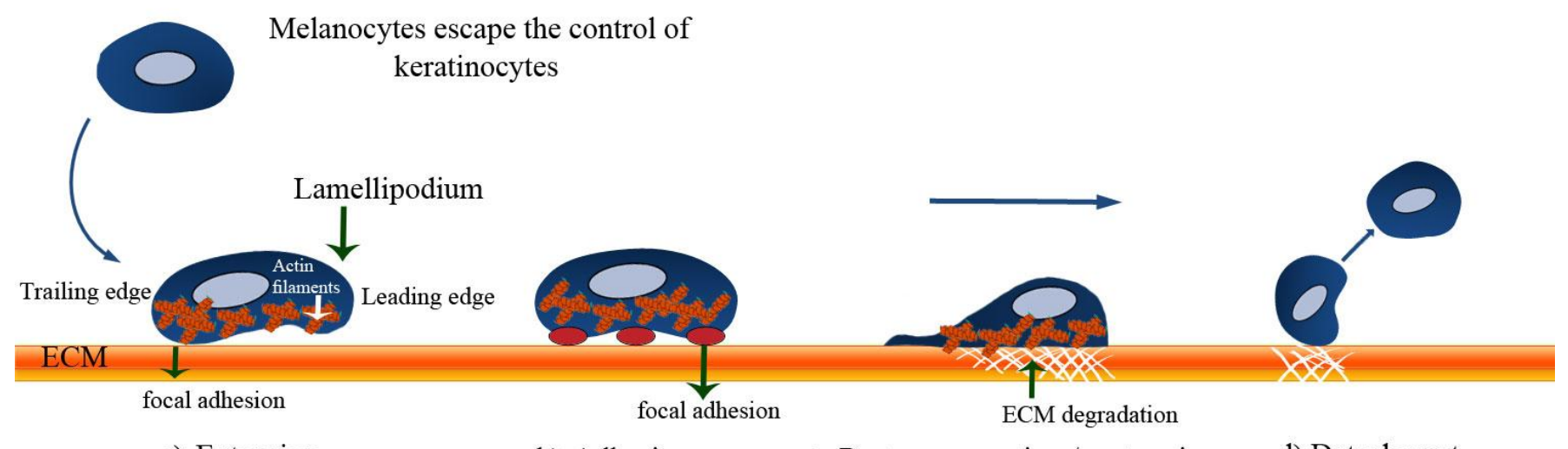
a) Extension
b) Adhesion

c) Protease secretion / contraction towards attachement site 
Figure 2. Molecular targets of antimigratory natural products.

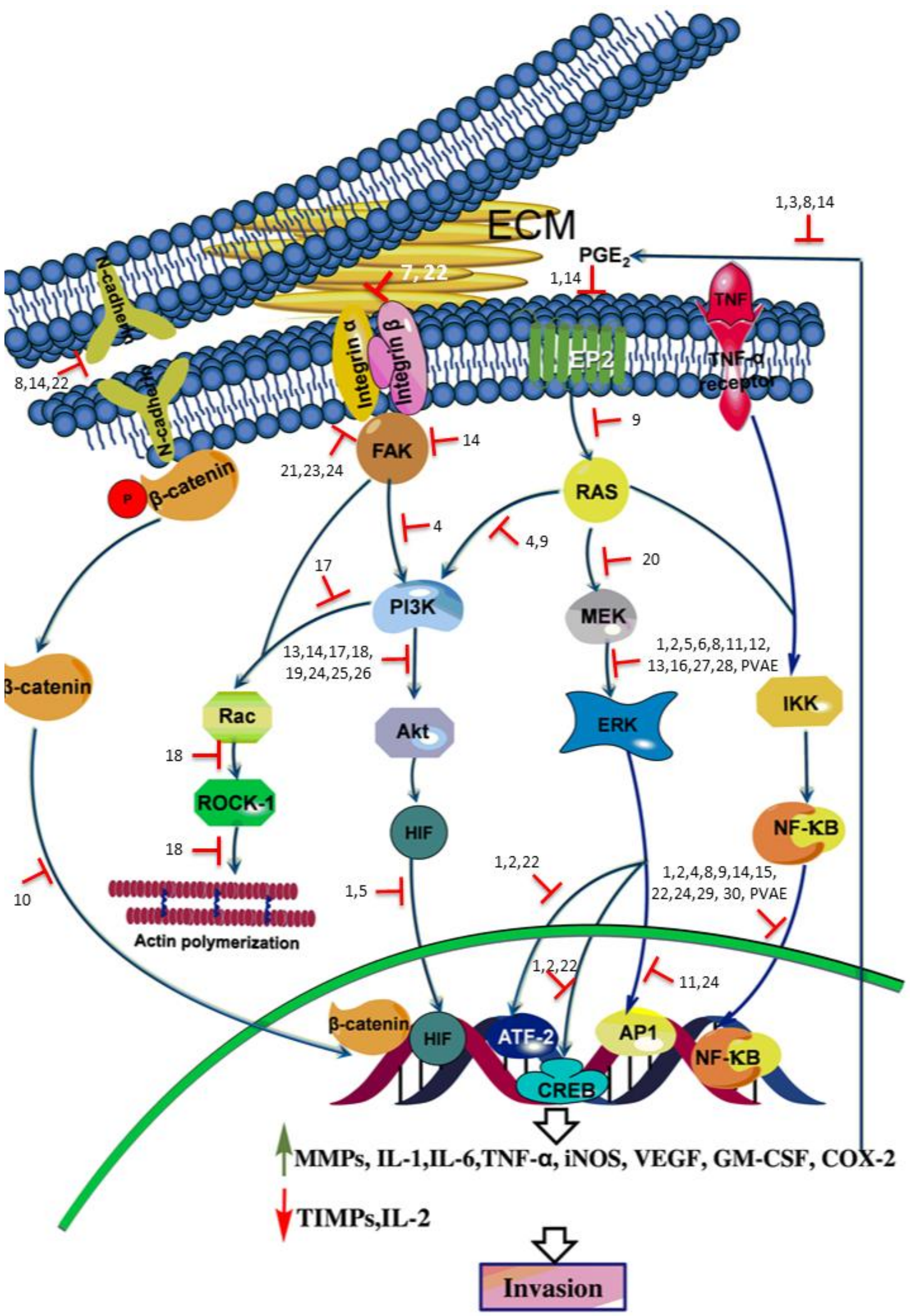

